\title{
Byzantine Fault-Tolerance with Commutative Commands
}

\author{
Pavel Raykov ${ }^{1}$, Nicolas Schiper ${ }^{2}$, and Fernando Pedone ${ }^{2}$ \\ 1 Swiss Federal Institute of Technology (ETH) \\ Zurich, Switzerland \\ 2 University of Lugano (USI) \\ Lugano, Switzerland
}

\begin{abstract}
State machine replication is a popular approach to increasing the availability of computer services. While it has been largely studied in the presence of crash-stop failures and malicious failures, all existing state machine replication protocols that provide byzantine faulttolerance implement some variant of atomic broadcast. In this context, this paper makes two contributions. First, it presents the first byzantine fault-tolerant generic broadcast protocol. Generic broadcast is more general than atomic broadcast, in that it allows applications to deliver commutative commands out of order - delivering a command out of order can be done in fewer communication steps than delivering a command in the same order. Second, the paper presents an efficient state machine replication protocol that tolerates byzantine failures. Our protocol requires fewer message delays than the best existing solutions under similar conditions. Moreover, processing of commutative commands on replicas requires only two MAC operations. The protocol is speculative in that it may rollback non-commutative commands.
\end{abstract}

\section{Introduction}

State machine replication is a popular approach to increasing the availability of computer services $[1,2]$. By replicating a service on multiple machines, hardware and software failures can be tolerated. Although state machine replication has been largely studied in the presence of crash-stop failures and malicious failures, all existing protocols that provide byzantine fault-tolerance (BFT) (e.g., [3-7]) implement some variant of atomic broadcast, a group communication primitive that guarantees agreement on the set of commands delivered and on their order. In this context, this paper makes two contributions.

The first contribution of this paper is a byzantine fault-tolerant generic broadcast protocol. Generic broadcast defines a conflict relation on messages, or commands, and only orders messages that conflict. Two messages conflict if their associated commands do not commute. For instance, two increment operations of some variable $x$ commute since the final value of $x$ is independent of the execution order of these operations. Generic broadcast generalizes atomic broadcast - the two problems are equivalent when every two messages conflict. Previous generic 
broadcast protocols appeared in the crash-stop model [8-10]; ours is the first to tolerate malicious failures. The difficulty with generic broadcast stems from the need to deliver commutative commands in two communication delays and ensure that their delivery order, with respect to non-commutative commands, is the same at all correct processes. To address this challenge under byzantine failures we define Recovery Consensus, an abstraction that ensures proper ordering between conflicting and non-conflicting messages. The proposed protocol requires $n \geq 5 f+1$ replicas to tolerate $f$ byzantine failures. We use Recovery Consensus at the core of our generic broadcast protocol.

The second contribution of this paper is a state machine replication protocol that generalizes and improves current byzantine fault-tolerant state machine replication protocols. Our protocol builds on our generic broadcast algorithm. A naive implementation of state machine replication based on generic broadcast to propagate commands to servers would lead to a best latency of three communication delays. We rely on speculative execution to provide an efficient algorithm that executes commutative commands in two communication delays. The algorithm is speculative in that it may rollback commands in some cases (i.e., when non-commutative commands are issued). To summarize, the principal advantage of the proposed state machine replication protocol is to allow fast execution of commutative commands in two message delays. Moreover, when commands commute servers only need to execute two MAC operations per command.

The remainder of the paper is structured as follows. Section 2 defines the system model. Sections 3 and 4, respectively, present the Recovery Consensus and generic broadcast protocols. We extend our generic broadcast protocol to provide state machine replication in Section 5. Section 6 discusses related work and Section 7 concludes the paper. Correctness proofs of the protocols can be found in the appendix of the full version of this paper [11].

\section{System model and definitions}

We consider an asynchronous message passing system composed of $n$ processes $\Pi=\left\{p_{1}, \ldots, p_{n}\right\}$, out of which $f$ are byzantine (i.e., they can behave arbitrarily). A process that is not byzantine is correct. The adversary that controls byzantine processes is computationally bounded (i.e., it cannot break cryptographic primitives) and cannot change the content of messages sent by one correct process to another correct process. The network is fully connected and quasi-reliable: if a correct process $p$ sends a message $m$ to a correct process $q$, then $q$ receives $m .^{3}$ We make use of public-key signatures to allow a process to sign a message $m$ [12]. We denote message $m$ signed by process $p_{i}$ as $\langle m\rangle_{\sigma_{i}}$. We also use HMACs [13] to establish a bidirectional authenticated channel between any two processes $p_{x}$ and $p_{y}$, with the notation $\langle m\rangle_{\sigma_{x y}}$ indicating a message $m$ signed with a secret key shared between processes $p_{x}$ and $p_{y}$.

\footnotetext{
3 The presented algorithms can trivially be modified to tolerate fair-lossy links, links that may drop messages but guarantee delivery of a message $m$ if $m$ is repeatedly sent. We assume quasi-reliable links to simplify the presentation of the algorithms.
} 
Due to the impossibility to solve consensus in asynchronous systems prone to crash failures [14], it is also impossible to solve atomic broadcast and generic broadcast [15]. This impossibility is typically overcome by strengthening the model with further assumptions (e.g., [16-18]). In this paper we assume the existence of an atomic broadcast oracle [19]. Atomic broadcast is defined by the primitives A-Bcast $(m)$ and A-Deliver $(m)$, where $m$ is a message. It guarantees the following properties:

- (Validity) If a correct process $p$ A-Bcasts a message $m$, then $p$ eventually A-Delivers $m$.

- (Agreement) If a correct process $p$ A-Delivers a message $m$, then every correct process $q$ eventually A-Delivers $m$.

- (Integrity) For any message $m$, every correct process $p$ A-Delivers $m$ at most once.

- (Order) If correct processes $p$ and $q$ both A-Deliver messages $m$ and $m^{\prime}$, then $p$ and $q$ A-Deliver them in the same order.

\section{Recovery Consensus}

In this section we introduce Recovery Consensus, an abstraction used by generic broadcast to order messages whose associated commands do not commute, also denoted as conflicting messages. Below, we provide an implementation of Recovery Consensus that employs digital signatures for message authentication.

\subsection{Problem definition}

Recovery Consensus allows each process $p_{i}$ to propose a set of non-conflicting messages NCSet ${ }_{i}$ and a set of conflicting messages CSet $_{i}$. The set NCSet $t_{i}$ is called non-conflicting since every pair of messages in it does not conflict, and the set $C S e t_{i}$ is called conflicting since for every message $m \in C S e t_{i}$ there is a message $m^{\prime} \in N C S e t_{i}$ such that $m$ and $m^{\prime}$ conflict. It ensures agreement on a set of non-conflicting messages NCSet and on a set of conflicting messages CSet. Additionally, Recovery Consensus guarantees that if $n_{c h k}-f$ correct processes $p_{i}$ propose a message $m$, i.e., $m$ belongs to either $N_{C S e t}$ or $C S e t_{i}$, where $n_{c h k}$ is a parameter of the problem, $m$ will be part of either NCSet or CSet.

More formally, Recovery Consensus is defined by primitives proposeRC $\left(\mathrm{NCSSt}_{i}, \mathrm{CSet}_{i}\right)$ and $\operatorname{decideRC}\left(\mathrm{NCS}_{\mathrm{S}}, \mathrm{CSet}\right)$. Provided that every correct process $p_{i}$ invokes proposeRC $\left(\right.$ NCSet $\left._{i}, \mathrm{CSet}_{i}\right)$ and there are no conflicting messages in $\mathrm{NCSet}_{i}$, the following properties are guaranteed:

- (Termination) Every correct process eventually decides on some pair of message sets.

- (Agreement) If two correct processes decide on pairs of message sets $\left(\right.$ NCSet $_{1}$, $\left.\mathrm{CSet}_{1}\right)$ and $\left(\mathrm{NCSet}_{2}, \mathrm{CSet}_{2}\right)$, then $\mathrm{NCSet}_{1}=\mathrm{NCSet}_{2}$ and $C$ Set $_{1}=\mathrm{CSet}_{2}$.

- (Validity) If a correct process invokes decideRC $(N C S e t, C S e t)$, then: 1. NCSet $\cap C$ Set $=\varnothing$. 
2. If a message $m$ belongs to $n_{c h k}-f N_{\text {CSet }}$ sets of correct processes, then $m \in N C$ Set.

3. No two messages in NCSet conflict.

4. If a message $m$ is in $n_{c h k}-f$ sets $N C S e t_{i} \cup C$ Set $_{i}$ of correct processes, then $m \in N C S e t \cup C$ Set.

\subsection{Solving Recovery Consensus}

Algorithm $\mathcal{C}_{a b s i g n}$ requires at least $n_{c h k}$ correct processes, where $n_{c h k} \leq n-$ $f$. It consists of a single task and works as follows. Each process $p_{i}$ starts by atomically broadcasting the pair $\left(N_{C S e t}, C_{i} S e t_{i}\right)$ signed with $p_{i}$ 's signaturein the algorithm, the signed message is denoted as $\left\langle\operatorname{NCSet}_{i}, \mathrm{CSet}_{i}\right\rangle_{\sigma_{i}}$ (line 2). Process $p_{i}$ then waits until it A-Delivered $n_{c h k}$ unique and valid messages, that is, messages from distinct sources that do not contain conflicting messages in NCSet $_{j}$ (line 3). Detecting unique messages is done with signatures: if $p_{i} \mathrm{~A}$ Delivers two messages from the same source $p_{j}$, then $p_{i}$ discards both messages since $p_{j}$ is byzantine. By considering the first $n_{c h k}$ unique and valid messages, this ensures that at least $n_{c h k}-f$ messages A-Delivered by $p_{i}$ were broadcast by correct processes.

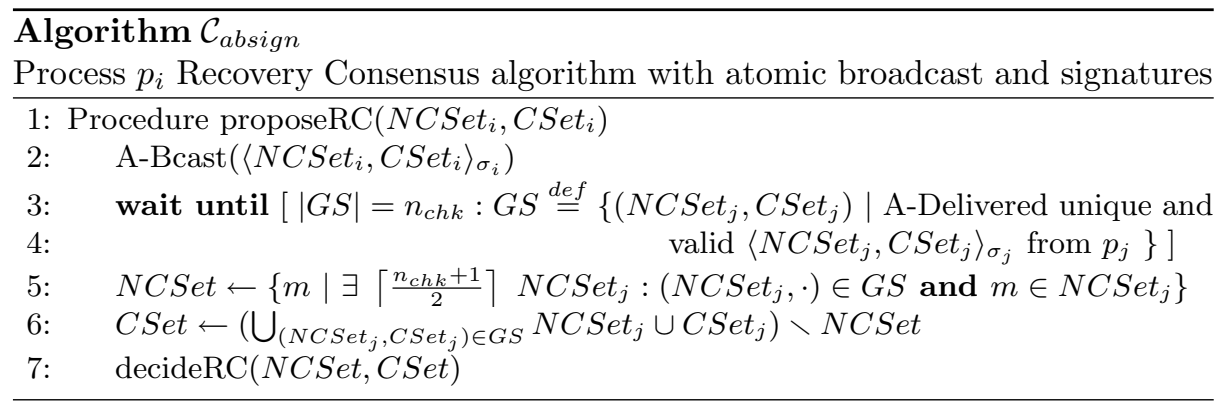

Any message that appears in a majority of the $n_{c h k} N C S e t_{j}$ sets will appear in NCSet (line 5). This guarantees the third validity property, namely that no two messages in NCSet conflict, since (i) the considered NCSet ${ }_{j}$ sets at line 3 do not contain conflicting messages and (ii) any message in NCSet belongs to a majority of $\mathrm{NCSet}_{j}$ sets.

Let $Q_{n_{c h k}-f}$ be a quorum of $n_{c h k}-f$ correct processes that propose a message $m$ as part of $N_{C S e t}$, and let $Q_{n_{c h k}}$ be a quorum of $n_{c h k}$ processes, the number of unique and valid A-Delivered messages processes consider at line 3. To ensure that we include $m$ in NCSet if $m$ belongs to $n_{c h k}-f N C S e t_{i}$ sets of correct processes, the minimum size of the intersection between $Q_{n_{c h k}-f}$ and $Q_{n_{c h k}}$ must be $\left\lceil\frac{n_{c h k}+1}{2}\right\rceil$. Hence, $n_{c h k}$ must satisfy inequality $\left(n_{c h k}-f\right)+n_{c h k} \geq n+\left\lceil\frac{n_{c h k}+1}{2}\right\rceil$. Since $n_{c h k} \leq n-f$, we conclude that $\mathcal{C}_{\text {absign }}$ requires $n>5 f$.

Finally, the set of conflicting messages $C S e t$ consists of messages gathered using atomic broadcast that are not part of NCSet (line 6). From the total order 
property of atomic broadcast, correct processes gather the same set of pairs $\left(N_{C S e t}, C_{j} S_{j}\right)$. Since NCSet and CSet are constructed from the gathered pairs using a deterministic procedure, all correct processes agree on these two sets.

\section{BFT Generic Broadcast}

We present a byzantine fault-tolerant generic broadcast protocol that we denote as $\mathcal{P G \mathcal { B }}$. This protocol relies on Recovery Consensus to handle conflicting messages. We first define generic broadcast in our model and then present the algorithm.

\subsection{Generic Broadcast}

Generic broadcast is defined by the primitives g-Broadcast $(m)$ and g-Deliver $(m)$, where $m$ is a message from the predefined set $\mathcal{M}$, to which all messages belong. We assume that each message broadcast has a unique identifier. Generic broadcast is parameterized by a symmetric relation $\sim$ on $\mathcal{M} \times \mathcal{M}$. If $\left(m, m^{\prime}\right) \in \sim$, or $m \sim m^{\prime}$ for short, we say that $m$ and $m^{\prime}$ conflict or are conflicting messages. If $m$ and $m^{\prime}$ conflict, then generic broadcast will order $m$ and $m^{\prime}$. If $m$ and $m^{\prime}$ do not conflict, they can be delivered in any order.

Generic broadcast guarantees the following properties, adapted from [10] to the byzantine failure model:

- (Validity) If a correct process $p$ g-Broadcasts a message $m$, then $p$ eventually g-Delivers $m$.

- (Agreement) If a correct process $p$ g-Delivers a message $m$, then every correct process $q$ eventually g-Delivers $m$.

- (Integrity) For any message $m$, every correct process $p$ g-Delivers $m$ at most once.

- (Order) If correct processes $p$ and $q$ both g-Deliver conflicting messages $m$ and $m^{\prime}\left(m \sim m^{\prime}\right)$, then $p$ and $q$ g-Deliver them in the same order.

As noted in [10], atomic broadcast is a special case of generic broadcast when all messages conflict with all messages, that is, $\sim=\mathcal{M} \times \mathcal{M}$. Thus, one could question the difficulty of implementing generic broadcast since we assume the existence of an atomic broadcast primitive that could be used to implement generic broadcast. The main idea of our generic broadcast protocol is that it allows fast delivery (i.e., in two communication delays) of non-conflicting messages, a bound that no atomic broadcast protocol can achieve in the general case [20].

\subsection{Solving Generic Broadcast}

The protocol is composed of two phases: an acknowledgment phase (ACK) and a check phase (CHK). Consecutive ACK and CHK phases form a "round". During the ACK phase processes g-Deliver non-conflicting messages in two message delays. 


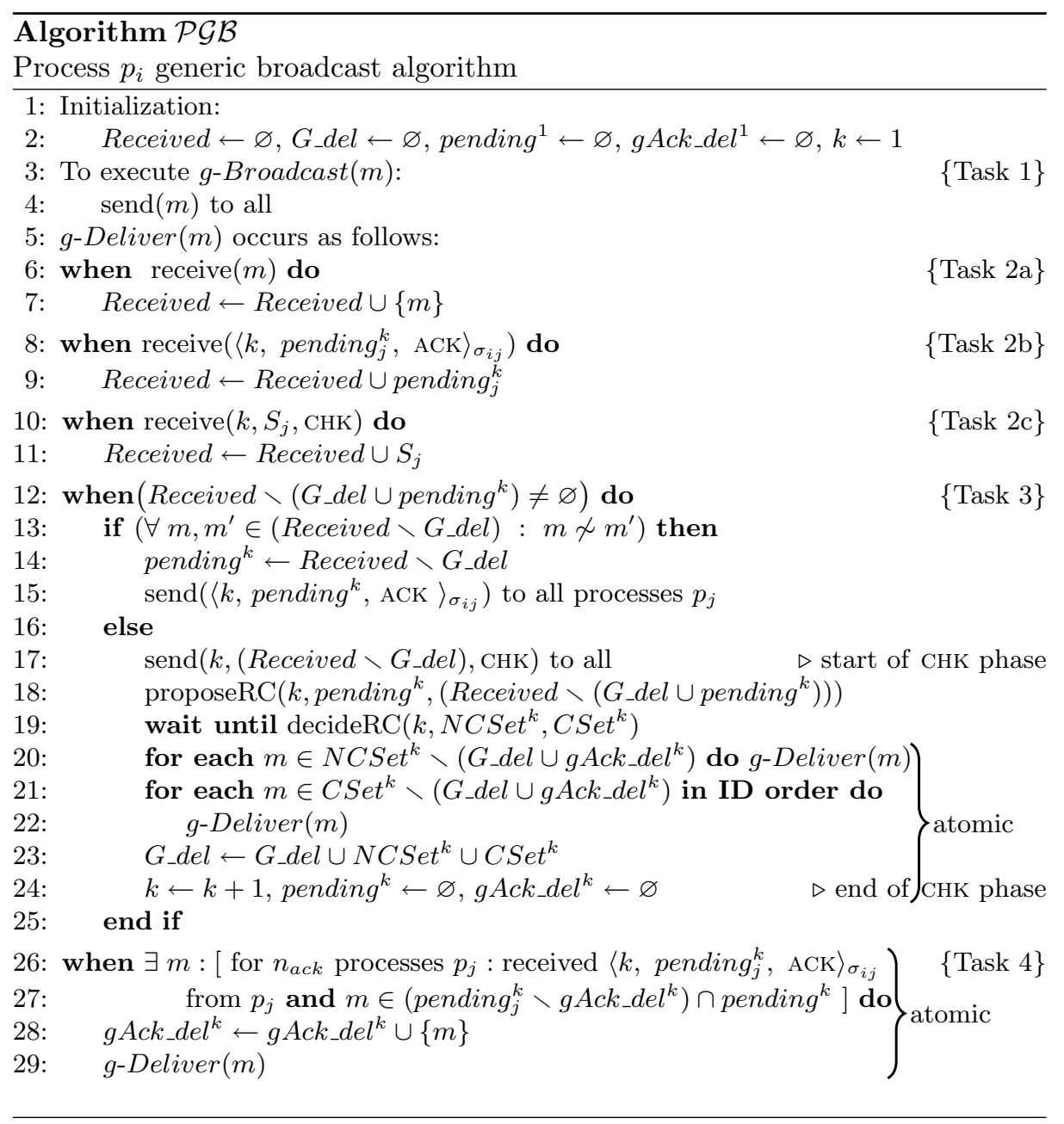

In the CHK phase, the protocol orders conflicting messages. Notice that $\mathcal{P G B}$ does not require signatures to deliver non-conflicting messages.

Algorithm $\mathcal{P G B}$ consists of six concurrent tasks. Each line of the algorithm, lines 20-24, and lines 26-29 are executed atomically. The following variables are used by the algorithm: $k$ defines the current round number, Received contains all the g-Broadcast messages that the process has received so far, $G_{-}$del contains all the messages that have been $g$-Delivered in the previous rounds, pending ${ }^{k}$ defines the set of non-conflicting messages acknowledged by the process in the current round, and $g A c k_{-} d e l^{k}$ is the set of messages g-Delivered in the ACK phase of the current round.

When a process $p$ wishes to g-Broadcast a message $m, p$ sends $m$ to all (line 4). When receiving $m$, a process $q$ adds $m$ to its Received set (line 7 ) and eventually checks whether $m$ conflicts with any message that was received but not 
delivered yet (line 13). If it is not the case, then $q$ adds $m$ to its pending ${ }^{k}$ set and acknowledges all messages in this set by sending pending ${ }^{k}$ to all (lines $\left.14-15\right) .{ }^{4} \mathrm{~A}$ process $q$ g-Delivers $m$ in the ACK phase when $q$ receives $n_{\text {ack }}$ acknowledgments for $m$ (lines 26-29). To prevent conflicting messages from being g-Delivered in the ACK phase despite $f$ byzantine processes, $n_{a c k}$ must be greater than $(n+f) / 2$.

It is possible that $q$ receives a message $m^{\prime}$ that conflicts with $m$ before receiving $n_{\text {ack }}$ acknowledgments for $m$. In that case, $q$ proceeds to the CHK phase. At this point, processes start by exchanging all messages that they received but did not deliver in previous rounds (line 17). In doing so, all correct processes eventually receive $m$ and $m^{\prime}$ despite potentially faulty senders, and enter the CHK phase. $\mathcal{P G B}$ then relies on Recovery Consensus to ensure agreement on the set of non-conflicting messages $N C S e t^{k}$ that were potentially g-Delivered in the ACK phase, and the set $C S e t^{k}$ of conflicting messages to deliver at the end of the current round. Correct processes invoke proposeRC with round number $k$, the set of non-conflicting messages pending ${ }^{k}$, and all the other messages that were received but not delivered so far, denoted as Received \(G_del $\cup$ pending $\left.{ }^{k}\right)$ (line 18), and decide on sets $N C S e t^{k}$ and $C_{S e t}{ }^{k}$ (line 19). Processes deliver non-conflicting messages in NCSet ${ }^{k}$ that they had not delivered so far (line 20), and then deliver conflicting messages $C_{\text {Set }}^{k}$ (line 22).

To ensure that if a message $m$ was delivered in the ACK phase $m$ will appear in set $N C S e t^{k}$ decided by Recovery Consensus, $m$ must be proposed by $n_{c h k}-f$ correct processes in pending ${ }^{k}$ at line 18. If $m$ was delivered in the ACK phase, at least $n_{\text {ack }}-f$ correct processes propose $m$ to Recovery Consensus. Hence, to maximize resilience we set $n_{a c k}$ equal to $n_{c h k}$.

Note that Recovery Consensus (Algorithm $\mathcal{C}_{\text {absign }}$ ) runs $n$ atomic broadcasts in parallel. Hence, when conflicting messages are issued, $\mathcal{P G B}$ has message complexity $n$ times bigger than a usual atomic broadcast protocol. $\mathcal{P G B}$ is optimized to perform well when non-conflicting messages are broadcast and Recovery Consensus is invoked rarely.

\section{State Machine Replication}

\subsection{A trivial algorithm}

Implementing state machine replication $[1,2]$ using the generic broadcast algorithm of Section 4 is straightforward: each command of the state machine corresponds to a message in the set $\mathcal{M}$ and the conflict relation on messages is defined such that two messages conflict if and only if their associated commands do not commute. For instance, if replicas store bank accounts, two deposit commands on the same account commute since their execution order does not have an effect on the final state of the state machine nor on the respective outputs of these commands, which in this case only contain an acknowledgment that the

\footnotetext{
4 To ensure that messages are not acknowledged twice and improve the efficiency of the algorithm, processes can remember the set of messages that were acknowledged and only acknowledge them once.
} 


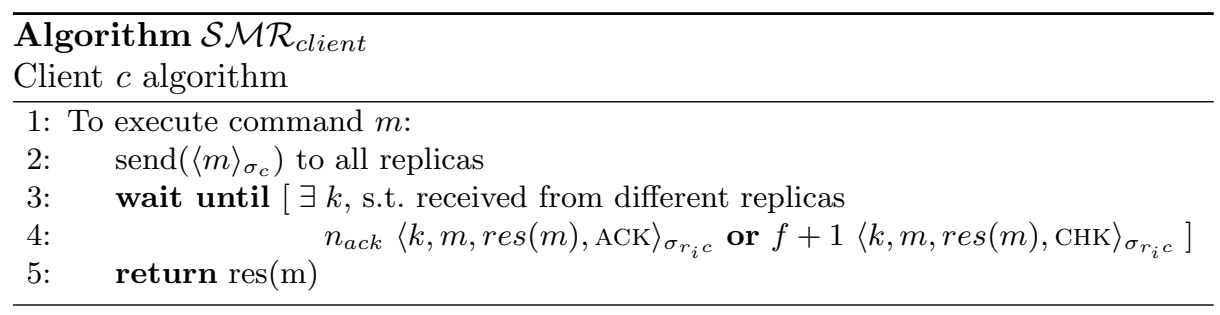

operations were successfully executed. Clients can then directly broadcast commands to replicas using Algorithm $\mathcal{P G} \mathcal{B}$. Once replicas deliver a command, they execute it and send back the result to the client. When $f+1$ identical replies are received by the client, the result of the command is known. This technique guarantees a form of linearizability [21,22].

\subsection{An optimal algorithm}

The above algorithm allows clients to learn the outcome of a command $\mathrm{cmd}$ in three communication delays if $\mathrm{cmd}$ commutes with concurrent commands. As we show next, a lower latency can be achieved by modifying Algorithm $\mathcal{P G B}$ and speculatively executing commands.

Before presenting the algorithm, we extend the system model of Section 2. We assume a population of $n$ replicas, aforenamed processes, and a set of clients. Any number of clients may be byzantine and $f$ bounds the number of faulty replicas. The latter execute a command $\mathrm{cmd}$ of the state machine by invoking execute $(\mathrm{cmd})$. This invocation modifies the state of the replica and returns a result. Commands are deterministic, that is, they produce a new state and a result only based on the current state. Our protocol speculatively executes commands and may require rolling back some commands if their speculative order does not correspond to their definitive order. The effect of operation rollback $(\mathrm{cmd})$ is such that if a sequence of commands $S e q$ is executed between execute $(\mathrm{cmd})$ and rollback $(\mathrm{cmd})$ then the replica's state is as if only commands in sequence $S e q$ were executed. Notice that although replicas may rollback some commands, clients always see the definitive result of a command (i.e., clients do not perform rollbacks).

The protocols for clients and replicas are presented in Algorithms $\mathcal{S} \mathcal{M} \mathcal{R}_{\text {client }}$ and $\mathcal{S} \mathcal{M} \mathcal{R}_{\text {replica }}$ respectively. The replica's algorithm is similar to $\mathcal{P G \mathcal { B }}$, except for the handling of acknowledgment messages, which is moved to the client. We highlight in gray the differences between $\mathcal{S} \mathcal{M} \mathcal{R}_{\text {replica }}$ and $\mathcal{P G \mathcal { B }}$. Similarly to $\mathcal{P G} \mathcal{B}$, replicas do not need to sign any messages when clients issue commutative commands.

When a client $c$ invokes a command $m, c$ sends $\langle m\rangle_{\sigma_{c}}$ to all replicas $\left(\mathcal{S} \mathcal{M} \mathcal{R}_{\text {client }}\right.$, line 2). A replica includes message $\langle m\rangle_{\sigma_{c}}$ in the Received set at lines 4,6,8 if $m$ 's signature is valid. When $m$ arrives at a replica $r$, one of two things can happen: either (a) $m$ does not conflict with any other command that 


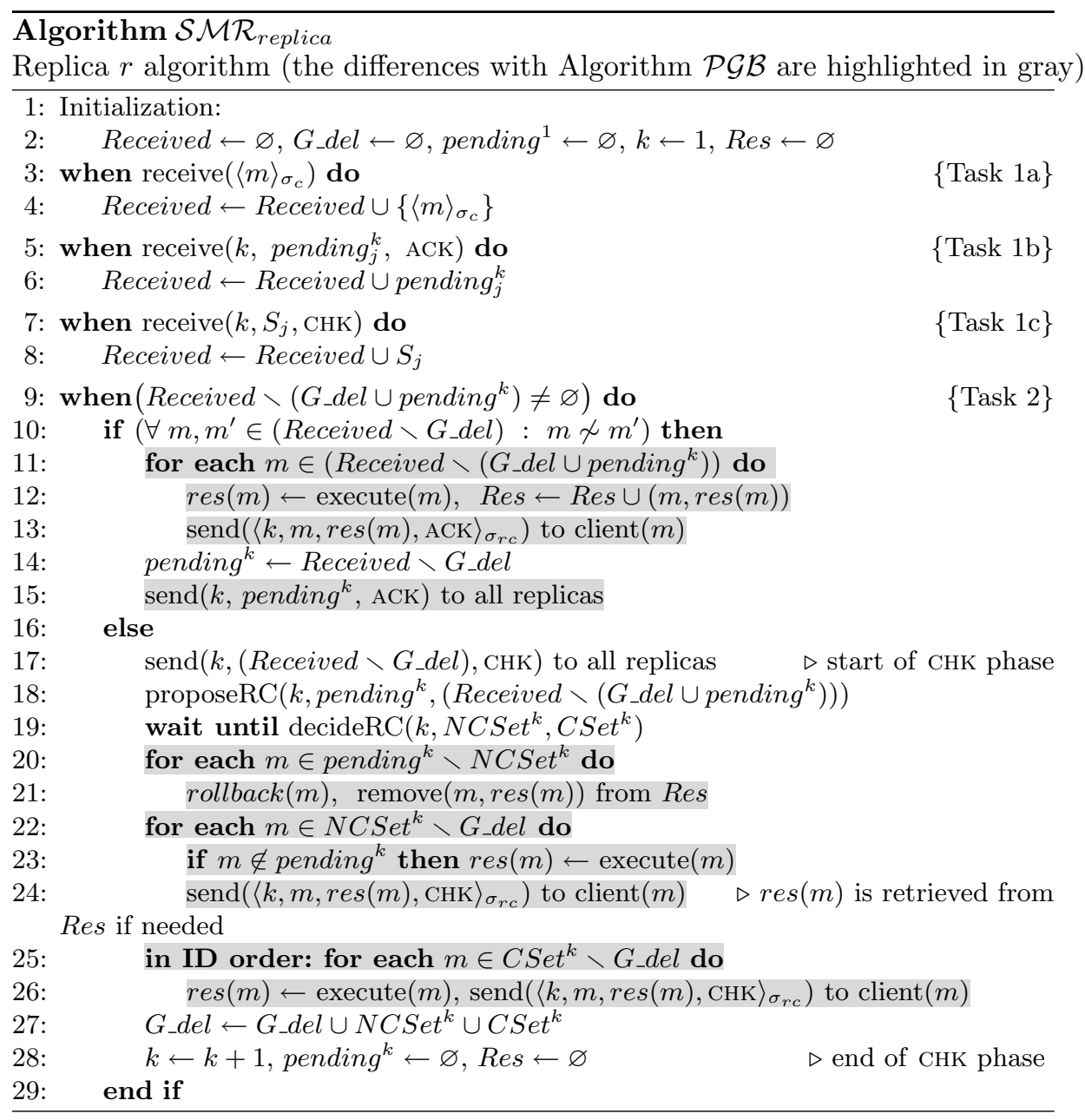

$r$ received in the current round or (b) $m$ conflicts with a command received in the same round.

In case (a), $r$ speculatively executes $m$, stores the result in set Res, and sends the result back to the client as an acknowledgment message $\left(\mathcal{S} \mathcal{M} \mathcal{R}_{\text {replica }}\right.$, lines 10-12). We use a function client $(m)$ defining for a given message $m$ the client that issued $m$. If client $c$ receives $n_{\text {ack }}$ identical acknowledgment messages for $m, c$ learns the result of command $m\left(\mathcal{S} \mathcal{M} \mathcal{R}_{\text {client }}\right.$, lines $\left.3-5\right)$ - this is a similar condition under which a process can g-Deliver a message in the ACK phase of Algorithm $\mathcal{P G B}$.

In case (b), command $m$ conflicts with a command received in the current round. Similarly to $\mathcal{P G \mathcal { B }}$, each replica $r$ uses Recovery Consensus to order these commands. For each command $m^{\prime}$ that was received by $r$ in the ACK phase but that does not appear in the decided NCSet, $r$ rollbacks $m^{\prime}$ and deletes 
the corresponding entry from Res - the speculative execution order of $m^{\prime}$ differs from its final execution order $\left(\mathcal{S} \mathcal{M} \mathcal{R}_{\text {replica }}\right.$, line 21$)$.

Then, commands in NCSet are executed if they were not acknowledged in the ACK phase, and the results of these commands are sent to the corresponding clients (lines 22-24). Similar actions are done for the conflicting commands of $C S e t$ (lines 25-26). A client learns the result of a command $m$ that was executed in the CHK phase after receiving $f+1$ identical replies for $m\left(\mathcal{S} \mathcal{M} \mathcal{R}_{\text {client }}\right.$, line 4$)$.

\subsection{Optimizations}

We briefly discuss two optimizations allowing Algorithms $\mathcal{S} \mathcal{M} \mathcal{R}_{\text {client }}$ and $\mathcal{S} \mathcal{M R}_{\text {replica }}$ (a) to achieve the optimal latency of two communication delays in executions without contention, defined next, and (b) to avoid message signing by clients.

No contention. Assume that pending sets contain the order in which commands were received and executed by the replicas; essentially, a pending set becomes a command sequence. We say that two pending sets conflict if they contain two conflicting messages executed in a different order. When no pending sets conflict, we say that there is no contention.

The main idea behind this optimization is that now we consider the conflicts between pending sets instead of the conflicts between individual messages. In the optimized Algorithm $\mathcal{S} \mathcal{M} \mathcal{R}_{\text {client }}$, a client $c$ learns the result res $(m)$ of the execution of command $m$ if: (1) $c$ received $n_{\text {ack }}$ non-conflicting pending sets with $\operatorname{res}(m)$ or $(2) c$ received $f+1$ CHK messages with res $(m)$. A replica enters the CHK phase if: (1) it has received two conflicting pending sets or (2) it has received a CHK message indicating that some other replica entered the CHK phase in the current round.

Since conflicting commands can be executed in the ACK phase, provided that they are executed in the same order, replicas include the execution order of commands in sets NCSet $i$ proposed to Recovery Consensus. Hence, the Recovery Consensus algorithm must be modified and NCSet essentially contains commands proposed by $\left\lceil\frac{n_{c h k}+1}{2}\right\rceil$ replicas $r_{i}$ as part of $N C S e t_{i}$, such that no two pending sets containing $m$ include two non-commutative commands $m_{1}$ and $m_{2}$ that were executed before $m$ and in different orders.

Avoiding message signing by clients. Digital signatures based on asymmetric cryptography can be expensive to generate or verify, let alone the problem of distributing and refreshing key pairs. Instead of signing a message $m$, clients can use an authenticator (a list of HMACs) to authenticate $m$ [5].

We modify Algorithm $\mathcal{S M R}_{\text {replica }}$ as follows: (1) during the ACK phase replica $r_{j}$ puts message $m$ at lines 4,6,8 in the Received set only if $m$ 's authenticator contains a valid HMAC entry for $r_{j}$; (2) during the CHK phase, we change the way $C S e t$ is built in the underlying protocol $\mathcal{C}_{\text {absign }}$ : message $m$ is included in CSet only if it belongs to $f+1$ different $N_{C S e t} \cup \operatorname{CSet}_{j}$. This 


\begin{tabular}{l|c|c|c|c|c|c} 
Protocol & PBFT [5] & Zyzzyva [3] & HQ [6] & Q/U [4] & Aliph [7] & this paper \\
\hline Resilience & $f<n / 3$ & $f<n / 3$ & $f<n / 3$ & $f<n / 5$ & $f<n / 3$ & $f<n / 5$ \\
\hline Best-case latency & 4 & 3 & 4 & 2 & 2 & 2 \\
\hline $\begin{array}{c}\text { Best-case latency } \\
\text { in the absence of... }\end{array}$ & bf & $\begin{array}{c}\text { bf } \\
\text { slow links }\end{array}$ & $\begin{array}{c}\text { bf } \\
\text { contention }\end{array}$ & $\begin{array}{c}\text { bf } \\
\text { contention }\end{array}$ & $\begin{array}{c}\text { bf } \\
\text { contention } \\
\text { slow links }\end{array}$ & $\begin{array}{c}\text { bf } \\
\text { contention }\end{array}$ \\
\hline $\begin{array}{c}\text { MAC operations at } \\
\text { bottleneck server }\end{array}$ & $2+8 \mathrm{f}$ & $2+3 \mathrm{f}$ & $2+4 \mathrm{f}$ & $2+4 \mathrm{f}$ & $2^{5}$ & 2 \\
\hline $\begin{array}{l}\text { Command classification } \\
\text { read-only/ } \\
\text { mutative }\end{array}$ & $\begin{array}{c}\text { read-only/ } \\
\text { mutative }\end{array}$ & $\begin{array}{c}\text { read-only/ } \\
\text { mutative }\end{array}$ & $\begin{array}{c}\text { read-only/ } \\
\text { mutative }\end{array}$ & none & $\begin{array}{c}\text { by conflict } \\
\text { relation } ~\end{array}$ \\
\hline Client-based recovery & no & yes & yes & yes & yes & no
\end{tabular}

Table 1. Byzantine fault-tolerant replication protocols ("bf": "byzantine failures").

guarantees that only client $c$ can issue commands with $c$ 's identifier, i.e., it is impossible to impersonate client $c$.

Unfortunately these modifications are more difficult to apply in Recovery Consensus. To avoid expensive signing during Recovery Consensus one could use matrix signatures [23] or employ the approach described in [6] for signing certificates, both of which essentially trade off signatures for additional network delays.

Digital signatures scale better than authenticators, whose size grows linearly with the number of replicas, so deciding which technique to apply depends on the specific system settings. In any case, we note that by design, Algorithms $\mathcal{S} \mathcal{M} \mathcal{R}_{\text {replica }}$ and $\mathcal{S} \mathcal{M} \mathcal{R}_{\text {client }}$ optimize the ACK phase, since this is the case we expect to happen more often.

\section{Related work}

In the following we compare our BFT state machine replication protocol to the related work (see Table 1). To the best of our knowledge, this paper is the first to present an implementation of byzantine generic broadcast. All BFT state machine replication protocols we are aware of have a "fast mode" - analogous to the ACK phase, where messages are delivered fast under certain assumptions (also called "best-case"), and a recovery mechanism to switch to a "slow mode" analogous to the CHK phase that resolves possible problems, usually contention or failures. Despite these similarities, existing protocols differ from each other in a number of aspects:

- PBFT was the first practical work on BFT state-machine replication. The best-case latency of four message delays is achieved when there are no byzantine failures. For read-only operations, the protocol can be optimized to achieve a latency of two message delays.

\footnotetext{
${ }^{5}$ In Table 2 of paper [7], Aliph's latency and throughput represent two different subprotocols: Chain and Quorum. We here show the number of MAC operations that Quorum uses since only Quorum achieves the best-case latency of two network delays.
} 
- Zyzzyva employs tentative execution to improve the best-case delay of PBFT. It executes commands in three network delays when there are no byzantine failures and links are timely. Otherwise, the protocol requires five network delays. Like PBFT, it can be optimized to execute read-only operations in two message delays.

- HQ, a descendant of PBFT, is optimized to execute read-only commands in two message delays and update commands in four message delays when the execution is contention-free.

- Q/U was the first protocol to achieve the best-case latency of two network delays for all commands when replicas are failure-free and updates do not access the same object concurrently.

- In [7], the authors propose a modular approach to build BFT services based on the concept of abstract instances. An abstract instance is a BFT replication protocol optimized for specific system conditions that can abort commands. In this context, the authors propose Aliph, a composition of three abstract instances: Quorum, Chain, and PBFT. Quorum is optimized for latency and allows command execution in two network delays when links are timely and the execution is contention- and failure-free. Chain, on the other hand, is optimized for throughput and achieves a latency of $f+2$ network delays when there are no failures.

The protocol presented in this paper is the first to achieve a latency of two network delays when the execution is failure-free but concurrent commutative commands are submitted. Under the same conditions, PBFT and Zyzzyva achieve latency of four and three message delays respectively, while $\mathrm{HQ}, \mathrm{Q} / \mathrm{U}$ and Aliph run an additional protocol to resolve contention.

$\mathrm{Q} / \mathrm{U}[4]$ and HQ [6] proposed a simplified version of the conflict relation: all commands are either reads or writes [6] (respectively, queries and updates in [4]); reads do not conflict with reads, and writes conflict with reads and writes. This is more restrictive than a conflict relation, as mutative operations on the same object do not necessarily conflict (e.g., incrementing a variable).

Zyzzyva, Q/U, and Aliph, more specifically the Quorum instance, do not use inter-replica communication to agree on the order of commands; instead they assume that it is the client's responsibility to resolve contention by collecting authenticated responses from replicas and distributing a valid certificate to the replicas. PBFT and the state machine replication protocol presented in this paper rely on inter-replica communication to serialize commands, which allows a lightweight protocol for clients. HQ uses a hybrid approach: it uses inter-replica communication only when clients demand to resolve contention explicitly, while in the "fast case" clients coordinate the execution.

Finally, we note that although [24] executes commutative commands in parallel, all commands are totally ordered using PBFT, resulting in a higher latency than our protocol in the aforementioned scenario. 


\section{$7 \quad$ Final remarks}

This paper introduces the first generic broadcast algorithm that tolerates byzantine failures. Generic broadcast is based on message conflicts, a notion that is more general than read and write operations $[4,6]$. The proposed algorithm is modular and relies on an abstraction called Recovery Consensus, used to ensure that correct processes (i) deliver the same set of non-conflicting messages in each round, and (ii) agree on the delivery order of conflicting messages. A modular approach facilitates the understanding of the ACK phase and CHK phase of Algorithm $\mathcal{P G B}$, and allows to explore various implementations of Recovery Consensus. We provided an implementation of Recovery Consensus that is based on atomic broadcast and digital signatures and requires at least $5 f+1$ processes. Finally, we extended the proposed generic broadcast algorithm to provide state machine replication. The resulting protocol, with its optimizations, can execute commands in two message delays under weaker assumptions than state-of-the-art algorithms.

The Aliph protocol [7] opened new directions in the development of state machine replication protocols: it is now possible to combine different protocols in one that switches through given implementations of state machine replication under certain policies to speed up the execution. Hence, it could be an interesting task to implement the protocol proposed in this paper as an Abstract instance (Generic) and see the behavior of the resulting algorithm (e.g., Generic-ChainQuorum-Backup).

\section{References}

1. Lamport, L.: Time, clocks, and the ordering of events in a distributed system. Communications of the ACM 21 (1978) 558-565

2. Schneider, F.B.: Implementing fault-tolerant services using the state machine approach: A tutorial. 22 (1990) 299-319

3. Kotla, R., Alvisi, L., Dahlin, M., Clement, A., Wong, E.: Zyzzyva: Speculative byzantine fault tolerance. ACM Transactions on Computer Systems 27 (2009) 1-39

4. Abd-El-Malek, M., Ganger, G.R., Goodson, G.R., Reiter, M.K., Wylie, J.J.: Faultscalable byzantine fault-tolerant services. In: SOSP '05: Proceedings of the twentieth ACM symposium on Operating systems principles, New York, NY, USA, ACM (2005) 59-74

5. Castro, M., Liskov, B.: Practical byzantine fault tolerance and proactive recovery. ACM Transactions on Computer Systems 20 (2002) 398-461

6. Cowling, J., Myers, D., Liskov, B., Rodrigues, R., Shrira, L.: HQ replication: a hybrid quorum protocol for byzantine fault tolerance. In: OSDI '06: Proceedings of the 7th symposium on Operating systems design and implementation, Berkeley, CA, USA, USENIX Association (2006) 177-190

7. Guerraoui, R., Knežević, N., Quéma, V., Vukolić, M.: The next 700 bft protocols. In: EuroSys '10: Proceedings of the 5th European conference on Computer systems, New York, NY, USA, ACM (2010) 363-376 
8. Aguilera, M., Delporte-Gallet, C., Fauconnier, H., Toueg, S.: Thrifty generic broadcast. In: Proceedings of DISC'00, Springer-Verlag (2000) 268-283

9. Lamport, L.: Generalized consensus and paxos. Technical report, Microsoft Research MSR-TR-2005-33 (2005)

10. Pedone, F., Schiper, A.: Handling message semantics with generic broadcast protocols. Distributed Computing 15 (2002) 97-107

11. Raykov, P., Schiper, N., Pedone, F.: Byzantine fault-tolerance with commutative commands. Technical report, University of Lugano, http://www.inf.usi.ch/faculty/pedone/Paper/2011/2011OPODIS-full.pdf (2011)

12. Rivest, R.L., Shamir, A., Adleman, L.: A method for obtaining digital signatures and public-key cryptosystems. Communications of the ACM 26 (1983) 96-99

13. Bellare, M., Canetti, R., Krawczyk, H.: Keying hash functions for message authentication. In: Proceedings of the 16th Annual International Cryptology Conference on Advances in Cryptology. CRYPTO '96, London, UK, Springer-Verlag (1996) $1-15$

14. Fischer, M., Lynch, N., Paterson, M.: Impossibility of distributed consensus with one faulty process. Journal of the ACM 32 (1985) 374-382

15. Chandra, T.D., Toueg, S.: Unreliable failure detectors for reliable distributed systems. Journal of the ACM 43 (1996) 225-267

16. Ben-Or, M.: Another advantage of free choice (extended abstract): Completely asynchronous agreement protocols. In: PODC '83: Proceedings of the second annual ACM symposium on Principles of distributed computing, New York, NY, USA, ACM (1983) 27-30

17. Dwork, C., Lynch, N., Stockmeyer, L.: Consensus in the presence of partial synchrony. Journal of the ACM 35 (1988) 288-323

18. Toueg, S.: Randomized byzantine agreements. In: PODC '84: Proceedings of the third annual ACM symposium on Principles of distributed computing, New York, NY, USA, ACM (1984) 163-178

19. Cachin, C., Kursawe, K., Petzold, F., Shoup, V.: Secure and efficient asynchronous broadcast protocols. In: Advances in Cryptology — CRYPTO 2001. Volume 2139 of Lecture Notes in Computer Science., Springer Berlin / Heidelberg (2001) 524541

20. Lamport, L.: Lower bounds for asynchronous consensus. Distributed Computing 19 (2006) 104-125

21. Herlihy, M.P., Wing, J.M.: Linearizability: a correctness condition for concurrent objects. ACM Trans. Program. Lang. Syst. 12 (1990) 463-492

22. Malkhi, D., Reiter, M., Lynch, N.: A correctness condition for memory shared by byzantine processes. Unpublished manuscript (1998)

23. Aiyer, A.S., Alvisi, L., Bazzi, R.A., Clement, A.: Matrix signatures: From macs to digital signatures in distributed systems. In: DISC '08: Proceedings of the 22nd international symposium on Distributed Computing, Berlin, Heidelberg, SpringerVerlag (2008) 16-31

24. Kotla, R., Dahlin, M.: High-throughput byzantine fault tolerance. In: International Conference on Dependable Systems and Networks (DSN). (2004)

\section{A. Correctness Proofs}

In the proofs, we assume that $n=5 f+1$ and $n_{a c k}=n_{c h k}=4 f+1$. Note that algorithms remain correct if $n>5 f+1$ and $n_{a c k}=n_{c h k}>4 f+1$, as long as $n_{a c k}=n_{c h k} \leq n-f$. 


\section{A.1 A proof of Algorithm $\mathcal{C}_{\text {absign }}$}

Lemma 1. \#NCSet I $_{i} G$ with conflicting messages.

Proof. Follows directly from the fact that we wait for valid messages at line 3.

Lemma 2. Set GS contains pairs (NCSet $\left.{ }_{i}, \mathrm{CSet}_{i}\right)$ from $n_{c h k}$ different processes.

Proof. Follows directly from the fact that we wait for unique messages at line 3 .

Proposition 1 (Termination) If all correct processes execute proposeRC(NCSet ${ }_{i}$, $\left.C S e t_{i}\right)$, then every correct process eventually decides on some pair of message sets.

Proof. To show termination we need to show that the condition at line 3 is eventually satisfied. From the validity property of atomic broadcast and since $n_{c h k}=n-f$, there is a time after which all correct processes A-Delivered $n_{c h k}$ messages that are unique and valid. Consequently, all correct processes eventually decide on some pair of message sets.

Proposition 2 (Agreement) If two correct processes decide on pairs of message sets $\left(\mathrm{NCSet}_{1}, \mathrm{CSet}_{1}\right)$ and $\left(\mathrm{NCSet}_{2}, \mathrm{CSet}_{2}\right)$, then $\mathrm{NCSet}_{1}=\mathrm{NCSet}_{2}$ and $C$ Set $_{1}=$ CSet $_{2}$.

Proof. Since atomic broadcast delivers messages in the same order at all correct processes, and properties valid and unique are checked deterministically and uniformly at all correct processes, processes form the same $G S$ variable at line 3 . Since NCSet and CSet are deterministic functions of GS, all correct processes decide on the same pair of message sets (NCSet, CSet).

Proposition 3 (Validity-1) If a correct process invokes decideRC(NCSet, CSet), then $N C$ Set $\cap C$ Set $=\varnothing$.

Proof. Follows immediately from the definition of CSet at line 6 .

Proposition 4 (Validity-2) If a correct process invokes decideRC(NCSet, CSet) and a message $m$ belongs to $n_{c h k}-f N_{\text {NSet }}$ sets of correct processes, then $m \in N C S e t$.

Proof. At line 3 processes wait to receive sets $N_{C S} t_{i}$ from $n_{c h k}$ different processes. The minimum size of an intersection of two sets of processes of size $n_{c h k}$ and $n_{c h k}-f$ is at least $2 n_{c h k}-n-f$. So, if $m$ belongs to $n_{c h k}-f N_{\text {CSet }}$ sets of correct processes, $m$ appears in $2 n_{c h k}-n-f N C S e t_{i}$ sets in GS. Since $n=5 f+1$ and $n_{c h k}=4 f+1$, the size of the intersection is at least $2 f+1$, which corresponds to a majority $\left\lceil\frac{n_{c h k}+1}{2}\right\rceil$. Therefore, $m$ is in NCSet.

Proposition 5 (Validity-3) If a correct process invokes decideRC(NCSet, CSet), then no two messages in NCSet conflict. 
Proof. Suppose that the Lemma is false and there are two conflicting messages $m$ and $m^{\prime}$ that are present in the majority of the $N_{C S e} t_{i}$ sets belonging to set $G S$. This means that there is a $N C S e t_{i} \in G S$ such that $m, m^{\prime} \in N_{C S e t}$, a contradiction to Lemma 1 - there is a $\mathrm{NCSet}_{i} \in G S$ with conflicting messages.

Proposition 6 (Validity-4) If a correct process invokes decideRC(NCSet, CSet) and a message $m$ belongs to $n_{c h k}-f N_{C S e t} \cup C S e t_{i}$ sets of correct processes, then $m \in N C S e t \cup C$ Set.

Proof. The minimum size of an intersection of $n_{c h k}$ A-Delivered messages and $n_{c h k}-f$ correct processes who propose $m$ as part of NCSet $_{i} \cup C$ Set $_{i}$ is $2 n_{c h k}-$ $n-f$. Since $n=5 f+1$ and $n_{c h k}=4 f+1$, the size of this intersection is at least $2 f+1$. From lines 5 and $6 m \in C$ Set if $m$ does not already belong to NCSet. Therefore, $m \in N C$ Set $\cup$ CSet.

\section{A.2 A proof of Algorithm $\mathcal{P G B}$}

In the proofs below, we use the notation $G_{-} d_{e l}$, Received $_{x}, g A c k_{-} d e l_{x}^{k}$ to refer to the corresponding sets of process $p_{x}$. All processes progress by moving from round to round $(k=1,2, \ldots)$ or by staying in the same round forever. After executing the initialization at line 2 all correct processes enter the first round. The transition from round $k$ to $k+1$ occurs after the execution of the first atomic block (lines 20-24). All the lines and blocks marked as atomic are executed accordingly.

Lemma 3. If a correct process enters round $k$, then the $G \_d e l$ set of this process is not modified during round $k$.

Proof. Follows immediately from the fact that we update $G \_d e l$ set only at line 23 in the first atomic block.

Lemma 4. (1) If one correct process enters round $k$, then all correct processes enter round $k$; (2) at the beginning of every round $k$ the contents of $G_{-}$del sets are the same for all correct processes.

Proof. We prove this Lemma by induction on the round number $k$ :

(Base $\mathbf{k}=\mathbf{1}$ ) (1) If one correct process enters round 1, then all correct processes enter round 1 - this holds trivially, since all processes initially enter the first round.

(2) At the beginning of round 1 the contents of the $G_{-} d e l$ sets are the same at all correct processes - this is true since at the beginning of round 1 all processes have the same $G_{-} d e l$ set equal to $\varnothing$.

(Induction step $k \longrightarrow k+1$ ) To prove the induction step we need to show two statements:

(1) To show this we prove that all correct processes enter the CHK phase and terminate it in round $k$, if one correct process enters it. Suppose that one correct process $p_{x}$ entered the CHK phase in round $k$. It means that (i) there 
are two conflicting messages $m, m^{\prime} \in\left(\right.$ Received $_{x} \backslash G_{-}$del $\left.l_{x}\right)$ and (ii) $p_{x}$ executed line 17 sending Received ${ }_{x} \backslash G_{-}$del $_{x}$ to everyone. Let $p_{y}$ be any correct process $p_{y}$. We know that eventually $p_{y}$ receives message $\left(k,\left(\operatorname{Received}_{x} \backslash G_{-} d_{e l} l_{x}\right)\right.$, CHK $)$ from $p_{x}$. So, $p_{y}$ handles it at line 11 . Hence, we know that $m, m^{\prime} \in$ Received $_{y}$. By the induction hypothesis and Lemma $3 G_{-} d e l_{x}=G_{-} d e l_{y}$ during round $k$, consequently, $m, m^{\prime} \in$ Received $_{y} \backslash G_{-}$del $_{y}$ when $p_{y}$ executes line 13 and the condition evaluates to false. Consequently, $p_{y}$ enters the CHK phase. This shows that all correct processes enter the CHK phase and terminate it, a contradiction.

(2) By the induction hypothesis of 1 and 2, correct processes all terminate round $k$ if one enters it, and they all have the same sets $G_{-} d e l$ at the beginning of round $k$. Since all correct processes decide on the same pair $\left(N C S e t^{k}, C S e t^{k}\right)$ (due to the specification of Recovery Consensus in Section 3), after executing line 23 all correct processes have the same set $G \_d e l$.

Lemma 5. All correct processes g-Deliver the same set of messages in each round $k$. This set is $N C S e t^{k} \cup C S e t^{k}$, if a correct process executes line 23 in round $k$.

Proof. There are two cases to consider:

No correct process in round $k$ entered the CHK phase. Thus, it means that processes g-Delivered messages only by acquiring enough ACK messages at line 29 of Algorithm $\mathcal{P G B}$. So, suppose one correct process $p_{x}$ receives $n_{\text {ack }}$ pending sets with $m$. Since $n_{a c k}=4 f+1>f$, then there is some correct process $p_{y}$ that received $m$ and acknowledged $m$ in round $k$. Hence, $m \in$ Received $_{y} \backslash$ $G_{-} d e l_{y}$ and since all $G_{-} d e l$ sets are the same during round $k$ (by Lemmata 3 and 4$), m$ does not belong to set $G_{-}$del in round $k$. So, all correct processes eventually receive it, acknowledge it, and then g-Deliver it at line 29.

Some correct process in round $k$ entered the CHK phase. By Lemma 4 we know that if one correct enters the CHK phase in round $k$, then all correct processes will enter the CHK phase in round $k$. First, we show that for any correct process $p_{x}, g A c k_{-} d e l_{x}^{k} \subseteq N C S e t^{k}$.

Consider any $m \in g A c k_{-} d e l_{x}^{k}$. We know that $p_{x}$ received $n_{\text {ack }}$ acknowledgments for message $m$. So, there are at least $n_{a c k}-f$ correct processes $p_{j}$ with $m \in$ pending $g_{j}^{k}$. It means that if a correct process $p_{x}$ acknowledges message $m$ at line 15 and enters the CHK phase at line 18, then the input to Recovery Consensus pending $g_{x}^{k}$ at line 18 contains $m$. Hence, there are at least $n_{c h k}-f$ pending $g_{x}^{k}$ inputs with $m$ from correct processes to Recovery Consensus $\left(n_{a c k}=n_{c h k}\right.$ by assumption). By the specification of Recovery Consensus, $m$ appears in $N C S e t^{k}$. So, we have shown that $\forall m \in g A c k_{-} d e l_{x}^{k} \Longrightarrow m \in N C S e t^{k}$, so $g A c k_{-} d e l_{x}^{k} \subseteq N C S e t^{k}$. Second, we know that all correct processes eventually execute lines 20 and 22, consequently, all messages from $N C S e t^{k} \cup C S e t^{k}$ will be g-Delivered in the CHK phase of round $k$ (if they have not been g-Delivered before in the ACK phase of round $k$ ).

Proposition 7 (Validity) If a correct process $p$ g-Broadcasts a message $m$, then $p$ eventually g-Delivers $m$. 
Proof. Suppose $m$ is never g-Delivered by $p$. Since links are quasi-reliable and $p$ is correct, we conclude that all correct processes receive $m$ eventually. Let $k$ be the round in which the last correct process receives $m$. There are two cases to consider, either (1) no correct processes enter the CHK phase in round $k$, or (2) some correct process entered the CHK phase in round $k$.

We show that both cases lead to a contradiction:

In the first case, since no correct processes enter the CHK phase of round $k$, no correct processes received a message that conflicts with $m$ in round $k$. Hence, all correct processes acknowledge $m$, and $m$ is eventually g-Delivered by $p$, a contradiction.

Now consider the second case: by Lemma 4 all correct processes enter the CHK phase in round $k$. By assumption, all correct processes received $m$ by round $k$ and thus propose it to Recovery Consensus. Thus by the validity property of Recovery Consensus, message $m$ appears in NCSet ${ }^{k} \cup$ CSet $^{k}$. Consequently, $p$ g-Delivers $m$, a contradiction.

Proposition 8 (Agreement) If a correct process $p$ g-Delivers a message $m$, then every correct process $q$ eventually $g$-Delivers $m$.

Proof. If a correct process $p$ g-Delivers a message $m$ in some round $k$, by Lemma 4 , all correct processes enter round $k$. By Lemma 5 , all correct processes g-Deliver $m$ in round $k$.

Proposition 9 (Integrity) For any message $m$, every correct process $p$ g-Delivers $m$ at most once.

Proof. Message $m$ can be g-Delivered at the following lines:

At line 20. In this case, $m$ belongs to $N C S e t^{k}$, so $m$ cannot be delivered in the next line since $N C S e t \cap C S e t=\varnothing$. Message $m$ is then added to set $G_{-}$del in the same atomic block, hence $m$ cannot be delivered in any later round at lines 20 or 22 . Moreover, since $m$ belongs to set $G_{-} d e l, m$ cannot belong to the pending set of this process, hence be delivered in the ACK phase at line 29.

At line 22. In this case, $m$ appears in set $G_{-} d e l$ in the same atomic block. Similarly to the previous case $m$ cannot be delivered in later rounds.

At line 29. In this case, $m$ belongs to $g A c k_{-} d e l^{k}$ in some round $k$. In round $k, m$ cannot be delivered anymore in the ACK phase due to the condition of line 27. Message $m$ cannot be delivered at line 20 or line 22 either, since we explicitly deliver messages that do not belong to set $g A c k_{-} d e l^{k}$. At the end of the round, $m$ is added to set $G_{-} d e l$, thus $m$ cannot be delivered in later rounds for the same reasons as above.

Proposition 10 (Order) If correct processes $p$ and $q$ both $g$-Deliver conflicting messages $m$ and $m^{\prime}\left(m \sim m^{\prime}\right)$, then $p$ and $q g$-Deliver them in the same order.

Proof. If $p$ and $q$ g-Deliver $m$ and $m^{\prime}$ in different rounds, then the proposition is ensured by Lemma 5 and Proposition 9. Now suppose that $m$ and $m^{\prime}$ are delivered in the same round. There are three cases to consider: 
$p$ and $q$ g-Deliver $m$ and $m^{\prime}$ in the CHK phase: In this case, $m$ and $m^{\prime}$ have not been g-Delivered by $p$ and $q$ in the ACK phase. Consequently, either $m$ and $m^{\prime}$ will be g-Delivered by $p$ and $q$ in id order at line 22, or since $m \sim m^{\prime}$, one of this messages will be g-Delivered at line 20 and another will be g-Delivered at line 22 on both processes.

$p$ and $q$ g-Deliver $m$ and $m^{\prime}$ in the ACK phase: Since an intersection of two quorums of $n_{a c k}$ processes has at least one correct process $\left(2 n_{a c k}-n>f\right)$, messages $m$ and $m^{\prime}$ that conflict cannot be g-Delivered in the ACK phase.

$p$ g-Delivers $m$ in the ACK phase, and $q$ g-Delivers $m^{\prime}$ in the CHK phase (or vice-versa): Let $k$ be the round in which $m$ and $m^{\prime}$ are delivered. From Lemma $5, m$ belongs to the set $N_{C S} t^{k}$ decided by all correct processes with Recovery Consensus. Since $m \sim m^{\prime}$, from the specifications of Recovery Consensus: $m^{\prime} \notin N C S e t^{k}$. Since $q$ g-Delivers $m^{\prime}, m^{\prime} \in C_{S e t}{ }^{k}$. From the algorithm, $p$ g-Delivers $m$ followed by $m^{\prime}$, since $m^{\prime} \in C S e t^{k}$, and $q$ g-Delivers $m$ followed by $m^{\prime}$ since $m \in N C S e t^{k}$. The symmetrical case can be shown by simply exchanging occurrences of $p$ with $q$ and occurrences of $q$ with $p$.

\section{A.3 A proof sketch of Algorithms $\mathcal{S} \mathcal{M} \mathcal{R}_{\text {client }}$ and $\mathcal{S} \mathcal{M} \mathcal{R}_{\text {replica }}$}

We show that when replicas and clients respectively execute Algorithms $\mathcal{S} \mathcal{M} \mathcal{R}_{\text {replica }}$ and $\mathcal{S} \mathcal{M} \mathcal{R}_{\text {client }}$ the global execution history $H$ satisfies a form of linearizability [21] - the original definition in [21] does not work with byzantine clients.

Replicas progress by moving from round to round $(k=1,2, \ldots)$ or by staying in the same round forever. After executing the initialization at line 2 all correct replicas enter the first round. The transition from round $k$ to $k+1$ occurs after the execution of line 28. We define $H_{r}^{c m d}$ as the sequential execution history on replica $r$ up to the last command that does not commute with some command $c m d$. In $H_{r}^{c m d}$ we only consider the final execution of commands, that is, for any command $c m d^{\prime}$ in $H_{r}^{c m d}$, the final execution of $c m d^{\prime}$ is the one after which $c m d^{\prime}$ is not rollbacked anymore. Let $r_{1}$ and $r_{2}$ be two replicas. We say that histories $H_{c m d}^{r_{1}}$ and $H_{c m d}^{r_{2}}$ are equivalent if and only if (i) the histories contain the same set of commands and (ii) for each command $\mathrm{cmd}^{\prime}$ in the histories, the outputs of $c m d^{\prime}$ on $r_{1}$ and $r_{2}$ are the same, and the final states on $r_{1}$ and $r_{2}$ are the same.

Proposition 11 (Safety) For any correct replica $r$ and any command cmd that $r$ executes more than once, $r$ invokes rollback(cmd) exactly once between any two consecutive invocations of execute $(\mathrm{cmd})$.

Proof. Either the two consecutive invocations of execute $(\mathrm{cmd})$ are (a) in the same round or (b) not.

In case (a), from the properties of Recovery Consensus $N C S e t^{k} \cap$ CSet $^{k}=$ $\varnothing$, and thus cmd cannot be executed at lines 23 and 26. From lines 14 and 11, cmd cannot be executed twice at line 12 either. Moreover, if $\mathrm{cmd}$ is executed at line 12 , since $c m d \in$ pending $^{k}$, cmd cannot be executed at line 23 . Hence, the only possibility is that $\mathrm{cmd}$ is executed at lines 12 and 26 . In this case, $m$ belongs 


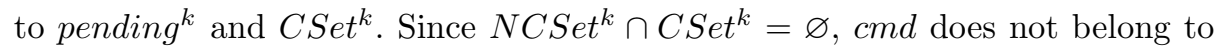
$N C S e t^{k}$, and $c m d$ is rollbacked once at line 21.

In case (b), if $\mathrm{cmd}$ is first executed at line 23 or $26, \mathrm{cmd}$ cannot be executed in any later rounds since $\mathrm{cmd}$ belongs to $G_{-}$del. If $\mathrm{cmd}$ is first executed at line $12 \mathrm{in}$ round $k$, since the second consecutive invocation of $\mathrm{cmd}$ occurs in round higher than $k, m$ does not belong to $N C S e t^{k}$. Hence, $c m d$ is rollbacked at line 21 in round $k$. Since commands are rollbacked only if they were previously executed at line $12, \mathrm{cmd}$ is not rollbacked again before its second execution.

Lemma 6. Let $C m d s_{r}^{k}$ be the set of commands executed in round $k$ on replica $r$ which are not rollbacked after their last execution in round $k$. For any two correct replicas $r_{1}$ and $r_{2}$ and any round $k, C m d s_{r_{1}}^{k}=C m d s_{r_{2}}^{k}$.

Proof. The proof is similar to Lemma 5.

Proposition 12 (Safety) For any two correct replicas $r_{1}$ and $r_{2}$ and any command cmd that $r_{1}$ and $r_{2}$ execute, $H_{r_{1}}^{c m d}$ is equivalent to $H_{r_{2}}^{c m d}$.

Proof. (i) From Proposition 11, each command that a correct replica executes is effectively executed once. Hence, from Lemma 6 , histories $H_{r_{1}}^{c m d}$ and $H_{r_{2}}^{c m d}$ contain the same set of commands.

(ii) For each command $c m d^{\prime}$ in the histories, the outputs of $c m d^{\prime}$ on $r_{1}$ and $r_{2}$ are the same since in each round (i) from Lemma 6 correct replicas execute the same set of commands and (ii) correct replicas execute commands that do not commute in the same order. For the same reason, the final states on $r_{1}$ and $r_{2}$ are the same after $r_{1}$ and $r_{2}$ respectively execute the sequence of commands $H_{r_{1}}^{c m d}$ and $H_{r_{2}}^{c m d}$.

Proposition 13 (Liveness) For any correct client c that issues a command cmd at line 2 of Algorithm $\mathcal{S} \mathcal{M} \mathcal{R}_{\text {client }}$, c eventually receives cmd's response at line 5.

Proof. Client $c$ receives $c m d$ 's response if $c$ receives (i) $n_{\text {ack }}$ ACK messages or (ii) $f+1$ CHK messages. We assume that (i) does not hold and show that (ii) holds. Since $c$ is correct and links are quasi-reliable, there exists a round in which $\mathrm{cmd}$ belongs to the Received set of all correct replicas - using a similar argument as in the proof of $\mathcal{P G B}$, we can show that if a correct replica enters a round $k$, then so do all correct replicas. If $\mathrm{cmd}$ has not already been decided in a Recovery Consensus instance of some round $k^{\prime}$, because $n=n_{c h k}+f$, there exists a round $k>k^{\prime}$ in which $n_{c h k}-f$ correct processes propose $c m d$ to Recovery Consensus as part of pending ${ }^{k}$ or Received $\backslash\left(G_{-}\right.$del $\cup$ pending $\left.{ }^{k}\right)$. Thus, from the fourth

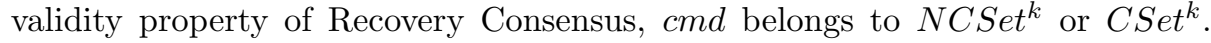
Since $n=5 f+1,4 f+1$ correct processes send a CHK message to $c$. Therefore, $c$ eventually receives $f+1$ CHK messages with $c m d$ 's response. 